\title{
Successful extracorporeal life support using a new micro-diagonal pump in a child with acute laryngotracheobronchitis, lung failure, and untractable septic shock
}

Thierry Carrel, MD, ${ }^{a}$ Erich Gygax, EBP, ${ }^{a} *$ Hans-Jürg Jenni, EBP, ${ }^{a}$ and Bendicht Wagner, MD, ${ }^{\mathrm{b}}$ Berne, Switzerland

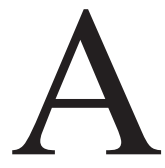

cute viral laryngotracheitis, the so-called "croup," is a widely known disease in pediatric medicine. Unless there are no signs of respiratory distress, children are treated ambulatory. Few of them require hospitalization and only $1 \%$ must be intubated because of respiratory failure. ${ }^{1}$ Bacterial pneumonia may complicate viral laryngotracheitis and adversely influence outcome. Even properly managed, viral laryngotracheitis can occasionally result in death, particularly in young infants. $^{2}$

\section{Clinical Summary}

A 14-month-old male infant $(8.7 \mathrm{~kg}, 80 \mathrm{~cm}$, body surface area $0.43 \mathrm{~m}^{2}$ ) had symptoms of a common cold but had to be hospitalized because of rapid deterioration of his general condition and severe respiratory distress syndrome. Despite treatment with oxygen, humidified air, inhalational epinephrine, and intravenous corticosteroids, he had to be intubated because of respiratory failure. Bilateral pleural effusions were drained. Oxygenation did not improve and conventional ventilation was switched to high-frequency oscillatory ventilation plus nitric oxide. Soon after, the child required increasing vasoactive support, and finally intractable septic shock and heart failure developed. Arterial saturation was between $70 \%$ and $78 \%$.

Extracorporeal circulatory support with oxygenation was found to be the last chance for this child. It was performed with a new micro-diagonal pump (DeltaStream DP-II; Medos, Stolberg, Germany). Cervical cannulation with the common carotid artery $(8 \mathrm{~F}$ cannula) and the internal jugular vein (14F cannula) was performed for vascular access.

Microbiologic assessment of sputum revealed parainfluenza and Staphylococcus aureus. Antibiotic regimen consisted of cefuroxime, amikacin, vancomycin, and clarithromycin.

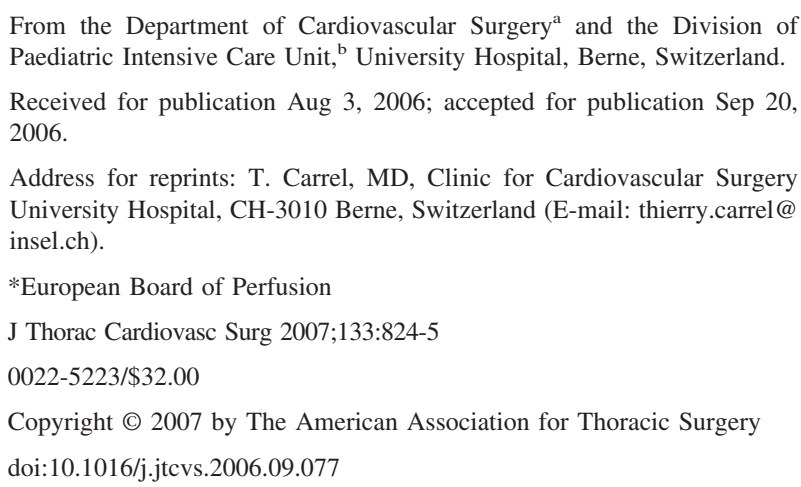

The DeltaStream DP2 diagonal pump is a mini-rotational pump with a diagonally streamed impeller (100$10,000 \mathrm{U} / \mathrm{min}$ ), which, by the decoupling of pump head and

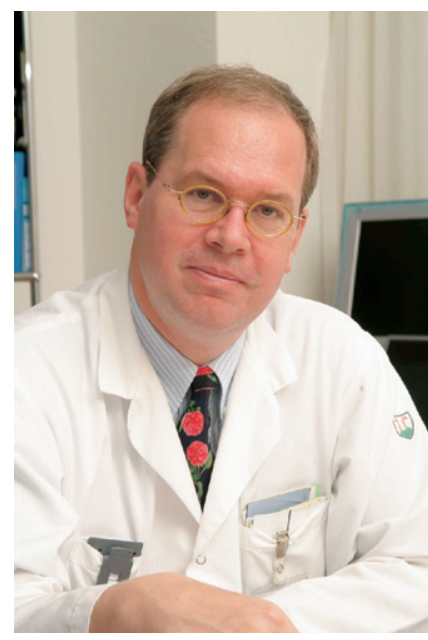

Dr Carrel drive, enables a nonpulsatile application comparable with a standard centrifugal pump (Figures 1 and 2). The DP2 pump (75 $\times 41 \mathrm{~mm}$ ) has an extremely low priming volume of $17 \mathrm{~mL}$. A variation of the impeller design has been performed to optimize hydraulic characteristics and reduce blood flow damage. The pump allows flow (up to $8 \mathrm{~L} / \mathrm{min}$ ) and pressure generation for cardiopulmonary bypass applications. ${ }^{3,4}$ The preload control prevents an aspiration of the cannula. The zero-flow mode allows direct interruption of the blood flow by reducing the rotational speed and so prevents an unintentional backflow. Additional safety features include a flow-measuring sensor with integrated bubble detector, four pressure sensors to be placed freely, as well as a level sensor for open cardiopulmonary bypass use.

Oxygenation was performed through a Polystan Safe Mini (Maquet, Hirrlingen, Germany) bioline-coated oygenator with integrated heat exchanger. Kaolin-activated anticlotting time was monitored every 2 hours with the HaemoTec system (Medtronic Inc, Englewood, NJ). Activated clotting time was targeted between 180 and 200 seconds.

\section{Discussion}

During the first 12 hours, extracorporeal membrane oxygenation (ECMO) was performed with a perfusion flow index of $2.8 \mathrm{~L} / \mathrm{m}^{2}$. Complete biventricular unloading favorably influenced the recovery of hypoxic/septic myocardial failure. Vasoactive medication was rapidly reduced and stopped.

Surprisingly, renal function did not deteriorate significantly during support (maximal serum creatinine $47 \mathrm{mg} / \mathrm{dL}$, urea 11.8 $\mathrm{mmol} / \mathrm{L}$ ). After 36 hours of full support, stepwise reduction of the arterial pump flow (200 mL/4 hours) allowed to increasingly load the heart and re-establish lung perfusion. Mechanical ventilation was restarted and pump flow could be stopped after 52 hours of support. No thrombembolic or bleeding events occurred during support. Platelet count was stable around 70,000 and only 1 unit of red blood cells and 1 unit of fresh frozen plasma were transfused.

Periodical clinical neurologic examination and continuous electroencephalographic monitoring were performed during ECMO support and did not show any abnormalities. On awaking, the child had focal contractions of the left hand and a discrete left-sided 

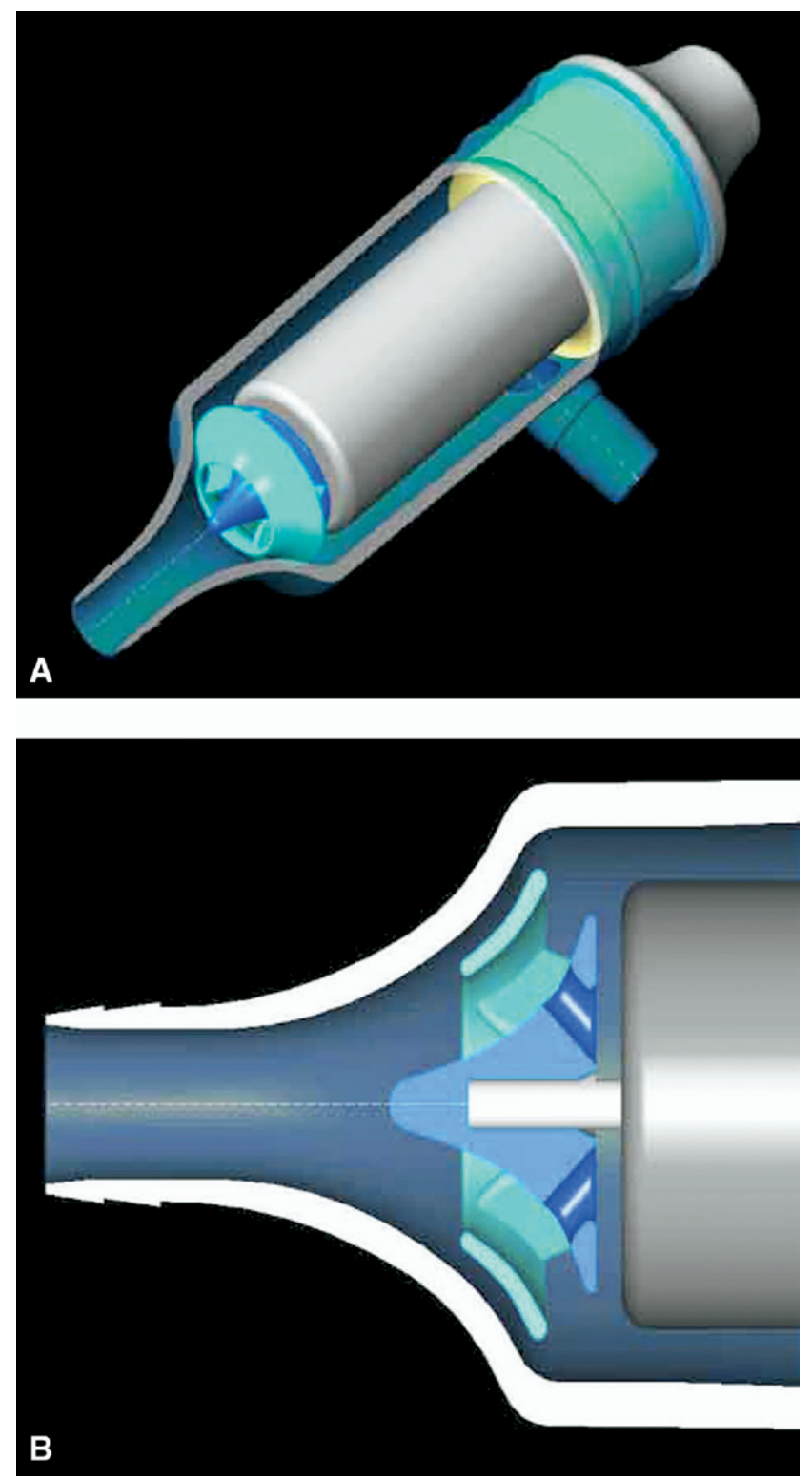

Figure 1. Line drawing of the DP 2 microdiagonal pump (A) showing the optimal washout mechanism due to the holes in the rotator (B).

hemiparesis. Cerebral computed tomographic scan revealed a small ischemic area in the right cortex. The child was extubated after 12 days and discharged 1 month after admission. At 1-year follow-up, the boy demonstrated a normal health status without neurologic residua.

ECMO support with the DP2 micro-diagonal pump allowed successful rescue of this dramatic case of laryngotracheobronchitis complicated by respiratory and circulatory failure.

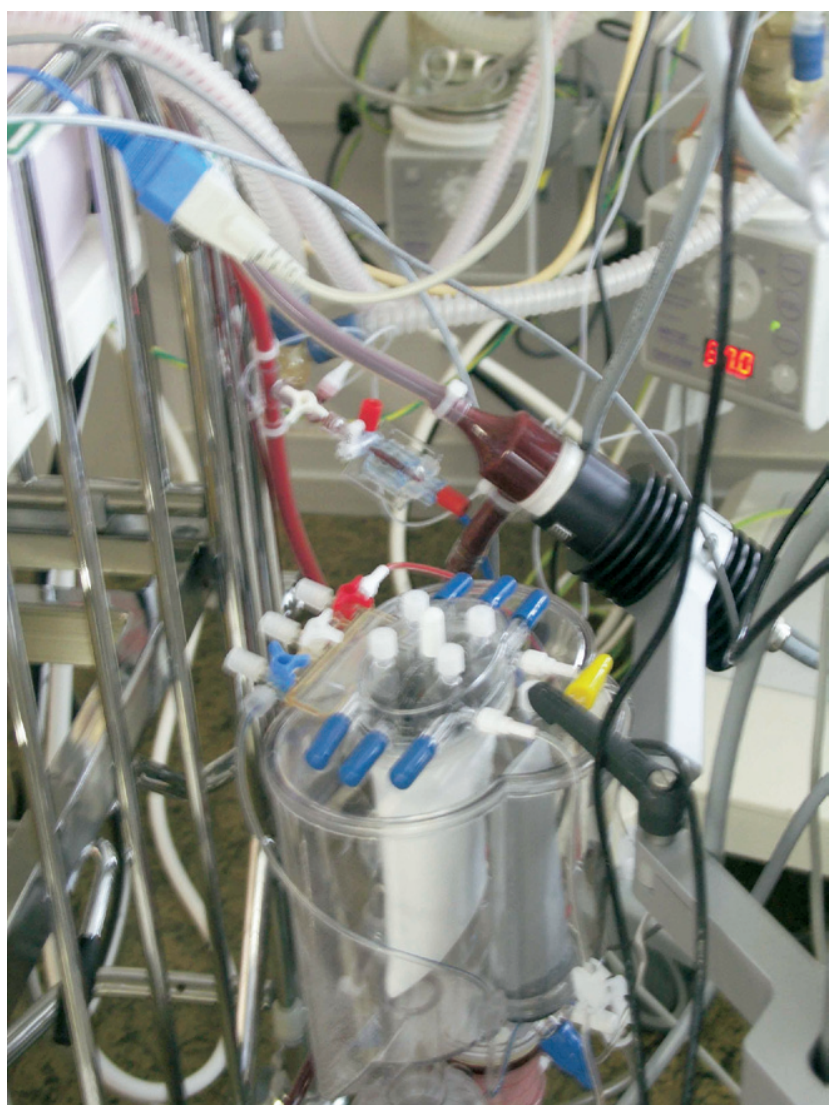

Figure 2. Picture of the extracorporeal circulatory and oxygenation system with the DeltaStream micro-diagonal pump.

The perfusion was performed without complications. The system heparin-coating of the oxygenator and the tubing system may have contributed to decrease procoagulant activity and need for heparin. ${ }^{5}$

\section{References}

1. Geelhoed GC. Sixteen years of croup in a Western Australian teaching hospital: effects of routine steroid treatment. Ann Emerg Med. 1996;28: 621-6.

2. Fisher JD. Out-of-hospital cardiopulmonary arrest in children with croup. Pediatr Emerg Care. 2004;20:35-6.

3. Agati S, Mignosa C, Ciccarello G, Dario S, Undar A. Pulsatile ECMO in neonates and infants: first European experience with a new device. ASAIO J. 2005;51:508-12.

4. Göbel C, Arvand A, Eilers R, Marseille O, Bals C, Meyns B, et al. Development of the MEDOS/HIA Delta Stream extracorporeal rotary blood pump. Artif Organs. 2001;25:358-65.

5. Cornish JD, Clark RH, Ricketts RR, Dykes FD, Wright JA, Kesser K, et al. Extracorporeal membrane oxygenation service at Egleston: two years experience. J Med Assoc. 1993;83:471-6. 